\title{
Estrategias metodológicas docentes en comprensión lectora y rendimiento académico en el área de Comunicación
}

\section{Methodological teaching strategies in reading comprehension and academic performance in the area of communication}

\section{Rosa Eufemia Choquichanca Lavado ${ }^{1}$ Miguel Gerardo Inga Arias ${ }^{2}$ \\ Universidad Nacional Mayor de San Marcos. Lima, Perú \\ Universidad Nacional Mayor de San Marcos. Lima, Perú \\ rositach2036@gmail.com \\ miguelinga.unmsm@gmail.com \\ ORCID 0000-0001-9251-6503 \\ ORCID 0000-0002-1588-0181}

Citar como: Choquichanca, R. e Inga, M. (2020). Estrategias metodológicas docentes en comprensión lectora y rendimiento académico en el área de Comunicación. Desde el Sur, 12(2), pp. 479-500.

\section{RESUMEN}

La base para el futuro educativo del estudiante es la educación temprana. Con esa premisa se implementaron el Programa Integral de Educación Temprana con Base en la Familia (Pietbaf), el Programa de Atención Integral de Grupos de Madres (Paigruma), las Salas de Educación Temprana (SET) y Cuna Más. Todos son importantes, pero se ha logrado muy poco, porque aún continúa la enseñanza tradicional. Por ello, el objetivo de esta investigación fue establecer si existe relación entre las estrategias metodológicas del docente en comprensión lectora y el rendimiento académico de los niños de 5 años en tres instituciones educativas (IE) de San Juan de Miraflores. Mediante el estadígrafo rho de Spearman encontramos que existe relación significativa positiva respecto a las estrategias de las dimensiones para la comprensión lectora, como los

1 Licenciada en Educación Primaria con segunda especialidad en Educación Inicial en la Universidad Nacional Enrique Guzmán y Valle. Docente de instituciones públicas de nivel inicial de la UGEL 1 de San Juan de Miraflores. Candidata a magíster en Educación con mención en Didáctica de la Comunicación en la Educación Básica por la Universidad Nacional Mayor de San Marcos.

2 Doctor en Educación, magíster en Lingüística y licenciado en Educación con mención en Lengua y Literatura por la Universidad Nacional Mayor de San Marcos. Docente investigador con el «Mérito Científico 2010». Articulista habitual en la revista de investigación del Instituto de Investigaciones de la Facultad de Educación. 
juegos de lenguaje, la lectura grupal en voz alta de cuentos infantiles, el teatrino, la observación-descripción-creación, la poesía y la expresión-imaginación-creatividad, y el rendimiento académico.

\section{PALABRAS CLAVE}

Estrategias docentes, comprensión lectora, rendimiento académico, 5 años

\section{ABSTRACT}

The basic foundation for all students' educational future is early education. That is why PIETBAF (Comprehensive Family-Based Early Education Program), PAIGRUMA (Comprehensive Care Program for Mothers' Groups), SET (Early Education Rooms) and CUNA MÁS were implemented. All of these programs are important; however, very little has actually been achieved because traditional teaching methods persist. In this context, the aim of this research was to establish whether or not a relationship exists between the methodological strategies employed by staff to teach reading comprehension and the academic performance of five-year-old children in three educational institutions in the Lima district of San Juan de Miraflores. Using Spearman's rank correlation coefficient, we found that there is a significant positive relationship between the strategies employed to develop reading comprehension (such as language games, reading aloud of children's stories in a group, puppet theater, observation-description-creation, poetry and expression-imagination-creativity) and academic performance.

\section{KEYWORDS}

Teaching strategies, reading comprehension, academic performance, five-year-old students

\section{Introducción}

En el Perú, luego de décadas marcadas por la falta de interés en la tarea pedagógica de desarrollar competencias en los niños, coherentes con la sociedad actual global moderna e informatizada, el Ministerio de Educación del Perú promovió y gestionó a nivel nacional mesas de trabajo para recoger la opinión de docentes, directivos, administrativos, autoridades y padres de familia, a fin de realizar los cambios que la educación inicial requiere y así mantener, direccionar y fortalecer el enfoque del Currículo 
Nacional anterior, además de tener docentes del área capacitados. Es imperativo que el nuevo marco pedagógico de educación inicial sea contrastado con la realidad: de qué modo los marcos teóricos y metodológicos de las competencias por áreas curriculares y los desempeños por niveles etarios son los oportunos. Para ello, es pertinente la capacitación docente, desde la lectura, la reflexión crítica y el buen uso de las estrategias para conocer las herramientas de espacio, material y rol del adulto en los niveles de desarrollo de las competencias y desempeños. Todos deben estar organizados por edad para una planificación, programación y evaluación desde el punto de vista formativo. En este proceso de contraste, la investigación se convierte en un instrumento científico de la bondad del nuevo diseño curricular en la formación inicial docente.

Por tanto, el objetivo central de este estudio fue investigar sobre la relación que existe entre las estrategias metodológicas docentes en comprensión lectora y el rendimiento académico en el área de Comunicación de los estudiantes de 5 años de instituciones educativas públicas del nivel inicial de la UGEL 1 de San Juan de Miraflores en Lima en 2019.

A lo largo de los años, nuestro país pasó por diversos cambios dentro del aspecto educativo. Algunos con un éxito mediano, otros con reformas que han perjudicado a los docentes $y$, por ende, a los estudiantes. Por ese motivo, el Perú necesitaba una mejora educativa de inmediato. Este proceso tomará aproximadamente 20 años, pero se debe iniciar antes de llegar al Bicentenario de la Independencia, para observar un cambio significativo. Si bien los programas de capacitación y actualización hacia los docentes fueron constantes en los últimos cinco años, tanto en las vacaciones de verano como en el año escolar, no resultaron suficientes. Los programas de educación temprana como el Pietbaf, el Paigruma, las SET y Cuna Más han sido fundamentales en el desarrollo de las capacidades del niño desde su infancia, porque han tratado de superar la situación de adversidad en la que se encuentra la educación de los niños en diferentes zonas del país.

De igual manera, las propuestas para solucionar las dificultades educativas tuvieron un gran avance. Sin embargo, no fue suficiente para la mejora en la educación. En la actualidad, esta situación se ve reflejada en las regiones del Perú. En los centros educativos, aún se continúa con la enseñanza tradicional y no se le otorga la importancia a la educación inicial. Se continúa con la desorganización y la ausencia de cobertura educativa, sabiendo que la intervención dentro de los primeros seis años de vida es fundamental para la educación y formación de la persona.

La educación inicial ha tenido una carencia en la eficacia de gestión por parte de las autoridades de los gobiernos de turno. Son necesarias la estimulación y la formación adecuada de los niños de 3 a 5 años, porque 
esto influye en su desarrollo educativo posterior. Esta etapa es el inicio de las bases para un buen desenvolvimiento futuro y el rol del docente es primordial para la construcción del niño como ciudadano. En el artículo de Amanda Rojas Bastard en el Congreso Mundial de Lecto-escritura en Valencia, se planteó lo siguiente:

Uno de los objetivos fundamentales de la Educación Preescolar lo constituye la preparación del niño para su posterior ingreso a la enseñanza primaria, fundamentalmente al primer grado. [...] en este proceso de adaptación a la nueva vida escolar desempeñan un rol fundamental tanto la maestra del grado preescolar y la de primer grado; la primera porque es la que culmina la etapa precedente, por tanto, es la llamada a realizar la preparación adecuada del niño para que esa transición ocurra de la forma más natural y menos traumática posible (Rojas, 2000, párr. 5).

El rol de la docente en la educación inicial es crucial. No obstante, en muchos centros educativos privados, se ejerce la enseñanza con docentes que no se encuentran capacitados e incluso con docentes de nivel primaria o secundaria. En otros casos no poseen el título profesional en el nivel de educación inicial. Lamentablemente, ante una escasez de la población docente en la especialidad inicial, el Ministerio de Educación, a través de la UGEL de cada sector, convocó a la contratación docente con título de nivel primario o secundario en una etapa extraordinaria de la adjudicación de contratos para ejercer la función de profesora de aula en el nivel inicial. Sin embargo, con la reforma educativa y la nueva Carrera Pública Magisterial, este aspecto se ha ido subsanando en los últimos cinco años. Las docentes de nivel inicial deben ser formadas en esa especialidad, ya sea en institutos pedagógicos o en universidades, para ejercer la función de profesora en las instituciones públicas, y las docentes con título profesional de nivel primario o secundario deben estudiar una segunda especialidad en el nivel inicial, además de tener experiencia laboral con un mínimo de tres años en el nivel inicial.

La regularización de este último aspecto aún no se aplica en su totalidad en algunas regiones inhóspitas y alejadas del país debido a la escasez de docentes. Asimismo, todavía existen algunas docentes que no garantizan un desempeño eficiente a causa de diversos factores, como la falta de una mejor preparación superior (estudios en instituciones educativas sin prestigio académico, universidades que no han sido licenciadas por Sunedu, falta de capacitación docente en competencias por parte del Estado o por recursos propios). 
El Diseño Curricular Básico Nacional (DCBN, 2010) da a conocer el informe del MED sobre los indicadores de la educación en el Perú realizado en 2006.

en las instituciones educativas del nivel inicial, públicas y privadas, viene laborando un gran número de docentes que no cuenta con el título requerido por la especialidad ( $27 \%$ en instituciones de educación inicial públicas y $51 \%$ en instituciones de gestión privada) [...] estos docentes [se desempeñan] en un nivel para el que no se formaron, esto es lo que el MED denomina docentes que no cumplen con el estándar de escolaridad (Minedu, 2010, p. 13).

Este aspecto se debió a la falta de seriedad por parte de las instituciones educativas de nivel pedagógico superior, ya que la mayoría de veces le otorgan importancia a un fin comercial o lucrativo por culminar los estudios de los estudiantes sin ningún rigor académico o profesional. Al detectar esta problemática se estableció que el diseño curricular que se generalizó a partir de 2000 se caracterizó por ser un currículo que introduce la investigación desde el inicio de la formación y propicia un contacto temprano del estudiante con la realidad a través de la práctica. Sin embargo, el estudio realizado por la Universidad ESAN señala que la investigación-acción se ha limitado a efectuar trabajos de tesis con fines de titulación y aspectos educativos en su mayor parte de poca utilidad social (Minedu, 2010, p. 10).

Por consiguiente, se crea una falsa expectativa, porque la docente supone que al egresar o titularse en aquellas instituciones educativas de nivel superior obtiene todo el bagaje y los conocimientos óptimos para aplicar y ejercer la carrera profesional. Son convocadas por instituciones de gestión privada a las que solo les interesa cubrir la vacante de docente sin importar la verdadera formación de los niños, y transforman a la educación como deficiente, ya sea en el área urbana o rural. Por otro lado, la Unidad de Medición de Calidad (UMC) que realiza la Evaluación Censal de Estudiantes (ECE) todos los años detecta problemas en la comprensión de textos en los niños que cursan el segundo grado de primaria.

A través del informe de la UMC se pudo identificar que el problema que presentan los estudiantes no solo se inicia desde su ingreso al nivel primario, ya que debieron ingresar al nivel escolar con toda la estimulación de su etapa de nivel inicial. Esta realidad demanda una mayor preparación por parte de los profesores en general y, en particular, de aquellos que se preparan en las instituciones de formación docente, de manera que puedan contribuir a la superación de las dificultades que presentan los alumnos tanto en zonas rurales como urbanas (Minedu, 2010, p. 11). De este modo, el rol del docente en la sociedad asume un protagonismo mayor y le exige 
estar capacitado para brindar los recursos necesarios en cuanto a la innovación y creatividad de los niños. Actualmente, en las instituciones educativas públicas mediante el director, los especialistas de la UGEL y los monitores, se supervisan las sesiones de aprendizaje en el aula. En cada visita observan, recogen las incidencias y luego proponen junto a la docente de aula una mejora en la planificación y ejecución de su sesión de aprendizaje.

Por otro lado, las evaluaciones internacionales reflejaron que los estudiantes no estuvieron preparados en un nivel óptimo en comprensión lectora y otras áreas. Esto se reflejó en la publicación del diario El Comercio, respecto a los resultados del Programa para la Evaluación Internacional de Estudiantes (PISA, por sus iniciales en inglés) de 2012.

En matemática, ciencia y comprensión lectora la realidad fue la misma. El Perú ocupó el último lugar entre los 65 países que participaron en el PISA de 2012. El examen de evaluación es elaborado cada tres años por la Organización para la Cooperación y el Desarrollo Económico (OCDE) (2013, párr. 1).

En el contexto nacional cuyos resultados se aproximan al ámbito de la investigación, se encuentra la prueba de Evaluación Censal de Estudiantes (ECE), que muestra el nivel de comprensión de lectora de los niños de segundo grado de educación primaria. Los resultados también fueron preocupantes, porque la mayoría de los estudiantes no entiende lo que lee.

Si se desea observar una verdadera transformación tanto física como cognitiva en los resultados de los niños de nivel primario, se deberá empezar por el desarrollo integral desde el nivel inicial, debido a que en esta etapa se les estimula para que se sientan capaces de socializar y sentir el placer por la lectura y ciencia. Si bien los niños de 5 años aún no saben leer, eso no es impedimento para que se familiaricen con los textos, ya que sus padres pueden colaborar con la lectura. Los niños logran inferir e interpretar la información que ha sido leída por sus padres. Así lo registró Rojas (2017, p. 8): «El estudiante construye el sentido del texto. Para ello, establece relaciones entre la información explícita e implícita». Los resultados se pueden evidenciar en el informe para la transferencia de gestión y reporte de cumplimiento misional de 2013-2016 del Ministerio de Educación, que publicó los avances en la educación del nivel inicial a 2017. Se expuso lo siguiente:

A fin de asegurar una adecuada oferta de docentes de nivel inicial, se han desarrollado acciones estratégicas tales como la implementación de programas de segunda especialidad en inicial a docentes de primaria y programas de formación profesional a docentes de inicial EIB sin título pedagógico y promotores educativos comunitarios de ámbitos amazónicos (Minedu, 2017, p. 16). 
De igual forma, estas acciones se evidenciaron en cifras como la publicación en el documento del Ministerio de Educación. Actualmente, están en marcha dos programas de formación docente en el nivel inicial: Segunda Especialidad y Formación EIB. El programa de formación en Segunda Especialidad está dirigido a 840 docentes de educación primaria que atienden servicios de educación inicial en ámbitos rurales (Cajamarca, La Libertad y Piura), mientras que el de Fortalecimiento de Capacidades en Gestión se dirige a 200 profesoras coordinadoras (Minedu, 2017, p. 16).

No obstante, la mayoría de veces los padres no están capacitados para estimular y educar a su hijo. Confunden el consentir a los niños con la formación en valores y responsabilidades en la casa y en la escuela. Por eso el Estado, por intermedio de los centros de estudios y los docentes, se comprometió en capacitar a las familias, para que funcione el binomio casa-escuela. Mediante la escuela para padres de familias o los talleres temáticos, se logró disminuir las debilidades presentadas en los resultados.

Por tanto, el presente estudio pretendió identificar la razón por la cual se presenta un bajo rendimiento en comprensión lectora en los niños, debido a que la lectura eficaz y su comprensión son la base para el aprendizaje de diferentes áreas del conocimiento. Esto resultará en el desarrollo de las habilidades cognitivas en el niño para el siguiente nivel escolar. Así lo plantea Rojas:

Dentro de los objetivos fundamentales en el primer grado se encuentra la enseñanza de la lectura, por lo que una de las tareas de la enseñanza preescolar es la de dotar al niño de una preparación que facilite el proceso de aprender a leer en el primer grado (Rojas, 2000, p. 3).

Se debe enfatizar que, en la preparación hacia la lectura del niño, este debe ponerse en contacto con el material escrito para que se familiarice y comprenda la función de la escritura. No es adecuado forzar hacia la lectura con historias o cuentos complejos. El niño sentirá la necesidad de leer por sí mismo tomándolo como algo placentero y recreativo. No hay que caer en la falsa idea de leer y leer en cantidad como en la enseñanza tradicional, donde se le daba importancia a la calificación más que a la comprensión. Así lo planteó Díaz y Trejo:

Leer no significa solo identificar un repertorio de signos que confirman un alfabeto y poder agruparlos en sílabas, palabras y frases, leer no es únicamente vocalizar letras. Leer es mucho más, leer es comprender, interpretar, descubrir y disfrutar. La comprensión de la lectura construye de nuevo imágenes, los sentimientos y los pensamientos que impregnan al lector en su propia subjetividad, lo que le da un toque personal al momento de leer (Díaz y Trejo, 2007, p. 14). 
Asimismo, en la etapa inicial, también denominada etapa preescolar, al niño se le debe estimular para que desarrolle su imaginación y creatividad mediante la lectura de cuentos infantiles, fábulas, historias regionales o locales, entre otras estrategias que deben emplear los docentes. Díaz y Trejo lo explicaron:

La escuela ha fallado en la tarea social, pues muchos estudiantes saben leer y entender literalmente el texto escrito, pero se les dificulta en ir más allá de lo textual para realizar una comprensión y valoración de lo leído, actividad que debe comenzar por estimular el placer de la lectura [aunque ellos] no lean por sí mismos (2007, p. 15).

Por lo tanto, esta investigación también se centró en la relación entre las estrategias metodológicas que utilizan las docentes para la mejora en la enseñanza y el rendimiento académico en la comprensión lectora de los niños de educación inicial de 5 años de las instituciones educativas públicas representativas de nivel inicial de la UGEL 1 de Lima Metropolitana: IEI 525 Reyna del Carmen, IE 652-29 Santa Rosa e IEI 516 Villa María.

Para definir las estrategias, se tuvo a Pozo (1996), quien señala que «las estrategias son procedimientos que se aplican de modo controlado, dentro de un plan diseñado deliberadamente con el fin de conseguir una meta fijada» (p. 299). En esta definición se enfatizó en los procedimientos que se deben seguir para lograr algo propuesto. Por otra parte, Solé (2011) señaló que las estrategias de comprensión lectora «son procedimientos de carácter elevado, que implican la presencia de objetivos que cumplir, la planificación de las acciones que se desencadenan para lograrlos, así como su evaluación y posible cambio» (p. 59). De acuerdo con ambos investigadores, se pudo definir que las estrategias de comprensión lectora son procedimientos, y que su uso ordenado y planificado conduce al logro de una meta u objetivo.

Entonces, es necesario que el docente planifique y utilice estrategias metodológicas de comprensión lectora para formar lectores autónomos, capaces de leer de manera inteligente diversos tipos de textos. Al respecto, algunos teóricos, como Solé (2011), precisaron que utilizar estrategias metodológicas de comprensión lectora contribuye a dotar a los estudiantes de recursos necesarios para aprender a aprender, que aprendan a leer mediante las intervenciones del docente, por lo que es necesario que asista a un proceso/modelo de lectura, que les permitan ver las estrategias de comprensión lectora en acción en una situación significativa y funcional.

También se puede mencionar a Condemarín (2000) y Cassany et al. (2007), quienes afirmaron la importancia de que los educadores conozcan y apliquen una serie de estrategias metodológicas de comprensión 
lectora destinadas a ayudar a los estudiantes a ser lectores competentes. Todos los autores citados coinciden en que el docente debe manejar y prever un conjunto de estrategias metodológicas de comprensión lectora que permitan a sus estudiantes comprender aquello que leen.

En ese sentido, para la nuestra investigación: las estrategias metodológicas de comprensión lectora se consideran un conjunto de procedimientos organizados que permiten al docente desarrollar la capacidad de comprensión de lectura de sus estudiantes. Estas estrategias metodológicas de comprensión lectora se usan antes, durante y después de la lectura, y deben estar presentes a lo largo de todo el proceso lector.

De igual forma, las docentes necesitan utilizar estrategias de aprendizaje que las ayuden a realizar una sesión de aprendizaje óptima en favor del proceso de comprensión lectora de los niños, como lo expresó Arellano:

Escribiremos acerca de los materiales literarios: la lectura escolar y su presencia en el currículo escolar, precisando que estos, por sí solos, no engendran la maravilla, sino que son los profesores y su intervención docente quienes los involucran en sus estrategias didácticas para que los alumnos gusten y aprendan de la lectura literaria (2012, p. 30).

En este contexto, la escuela debe organizar experiencias de aprendizaje en un medio que favorezcan la ampliación de ese conocimiento, posibilitando que los niños interactúen sistemáticamente con lectores experimentados que modelan el gusto por la lectura, tengan acceso a libros y bibliotecas, lean o se les lea diariamente, participen en conversaciones interesantes y significativas. El rol de la escuela es brindar todas las oportunidades para que los niños accedan a la enseñanza formal de la lectura de la manera más eficiente posible. Esta sería la clave para permitir un buen desarrollo lector posterior (Minedu, 2010, p. 7).

La lectura es una tarea cognitiva compleja que requiere de una enseñanza sistematizada. Así, la alfabetización temprana se ha convertido en un objetivo fundamental en los programas educativos de la mayoría de los países del mundo, y tienen como antecedente la importancia y la interacción entre los niños y los adultos potenciadores del lenguaje en todas sus formas. Estas estrategias no pueden ser formadas de una manera mecánica de aprendizaje seguida por reglas a cumplir, sino que deben apuntar a los objetivos de la actividad con la utilización de las estrategias para lograr que el niño se incentive y mejore su comprensión lectora.

deben existir elementos fundamentales en el uso de las estrategias, como la autodirección, que se define como la presencia y la conciencia de la existencia de un objeto: la planificación que tiene relación 
con la anticipación de los movimientos a seguir para el cumplimiento de un objeto; el autocontrol, que se refiere a la supervisión y evaluación de los propios elementos (Cáceres, 2012, p. 72).

Hay que tener en cuenta que las estrategias que se planteen no deben ser tomadas como un manual que se aplique de forma mecánica, rutinaria y precisa. Estas estrategias que se desarrollan deben estar planteadas de acuerdo con el contexto donde se va a desenvolver el profesor, quien será el orientador para elegir la mejor forma de aplicarlas y modificarlas, si fuera el caso, respecto a las situaciones que se presenten en el aula.

Las conexiones del texto con la propia vida se empiezan a estimular en el nivel inicial de 4 años. Se recomienda que las estrategias cognitivas para evocar imágenes sensoriales de lo que se va leyendo comience con lo siguiente:

Esta es también una actividad mental que se lleva a cabo mientras se va leyendo. Generar imágenes sensoriales significa imaginarse lo que se está leyendo, pero sin perder el hilo o la comprensión de lo que se sigue. Imaginarse el aroma, la imagen, el sentimiento, por ejemplo, de felicidad, dolor, pena. Se puede ayudar al alumnado a identificar en los pasajes «frases poderosas», frases que traen a la mente, de inmediato una imagen sensorial (Minedu, 2006, p. 37).

Leer es un proceso de interacción entre el lector y el texto. Un proceso mediante el cual el primero intenta satisfacer los objetivos que guían su lectura. El significado del texto se construye por parte del lector. La lectura es entendida como una habilidad cognitiva sumamente importante que permite al ser humano orientar su destino y buscar la verdad. Es una actitud mental y vital que desarrolla la emotividad y la inteligencia en procura de lograr en las personas sensibilidad para comprender su medio, transformar la realidad, reforzar la identidad y procurar el reencuentro de las personas consigo mismas y con su cultura.

Es así como el Ministerio de Educación otorgó a los docentes las Rutas de Aprendizaje, que fueron orientaciones pedagógicas y didácticas para una enseñanza efectiva y significativa. Uno de los fascículos comprende el área curricular de Comunicación del nivel inicial y presenta una competencia del área. Comprende textos escritos, lo cual nos explica detalladamente el proceso de comprensión en los niños:

- En esta competencia el estudiante comprende críticamente textos escritos de diverso tipo y complejidad en variadas situaciones comunicativas. Para ello, debe construir el significado de diversos textos escritos basándose en el propósito con que lo hace, en sus conocimientos, en sus experiencias previas y en el uso de estrategias específicas. Además, a partir de la recuperación de información explícita e inferida, y según 
la intención del emisor, evalúa y reflexiona para tomar una postura personal sobre lo leído.

- El estudiante localiza e identifica información que se presenta en el texto de manera explícita sin necesidad de hacer inferencias. A partir de esta, discrimina la que requiere según su interés y propósito.

- El estudiante asigna significado a los textos. Formula inferencias a partir de sus saberes previos, de los indicios que le ofrece el texto y del contexto en el que este se produce. Mientras va leyendo, verifica o reformula sus hipótesis de lectura.

- El estudiante toma distancia de las ideas propuestas en el texto o de los recursos utilizados para trasmitir ese significado, y juzga si son adecuados o no. Para ello, considera objetivamente el contenido y la forma, evalúa su calidad y adecuación con una perspectiva crítica. Opina reflexivamente sobre el texto usando argumentos que demuestren si lo comprendió.

- Uno de los objetivos más importantes en el nivel de educación inicial es conseguir que los niños disfruten de la lectura. Si bien no leen de manera convencional en esta etapa, sí pueden desarrollar un proceso activo al comprender lo que otros les leen, al coordinar lo que saben y sus experiencias con lo que ofrece el texto.

Por un lado, el objetivo principal de la comprensión lectora es que el estudiante asuma la lectura como una actividad relevante en su vida cotidiana. Para ello, se requiere lograr la transacción entre el lector y el texto, adquirir estrategias básicas para la comprensión, reconocer las técnicas que se aplican en diversos géneros de textos, distinguir y analizar la información relevante en los textos, aumentar el dominio del conocimiento específico de los textos, elaborar sus propios textos, reconocer la importancia de la lectura en nuestra sociedad y disfrutar de las diversas lecturas a las que se expone.

Por otro lado, el objetivo de la educación no es únicamente el compendio y la memorización de información, sino que esos conocimientos adquiridos conformen un aprendizaje significativo, de manera que puedan ser empleados y utilizados en diferentes situaciones de la vida cotidiana (Matesanz, 2013).

Morán y Uzcátegui (2006) señalaron que las estrategias de aprendizaje son «procedimientos (conjunto de pasos, operaciones o habilidades) que un aprendiz emplea en forma consciente, controlada e intencional como instrumentos flexibles para aprender significativamente y solucionar problemas». Por su parte, Peña (2000, citado por Morán y Uzcátegui, 2006) definió a las estrategias cognitivas como planes o programas estructurados para lograr un objetivo. Los rasgos más característicos de las estrategias de aprendizaje son tres. Primero, la autodirección, que comprende la 
fijación de un objetivo y la conciencia de que este existe. A tal fin, se parte de una toma de decisión y una planificación. Segundo, el autocontrol, que comprende la supervisión y evaluación del propio conocimiento, lo cual requiere de la aplicación del conocimiento metacognitivo. Y tercero, la flexibilidad, que permite imprimirle modificaciones a ese conocimiento si fuese necesario (Morán y Uzcátegui, 2006).

\section{Materiales y método}

Esta investigación contribuyó con un estudio de tipo descriptivo, correlacional, causal y empírico. Por el estudio de variables, fue bivariada y cuantitativa. Finalmente, por el tiempo de aplicación fue transversal o sincrónico.

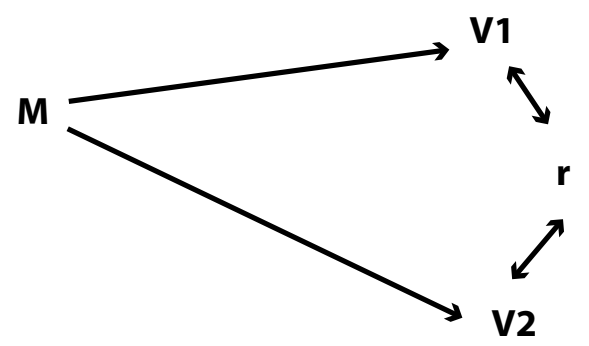

Donde:

$M=$ Muestra

$\mathrm{V} 1$ = Estrategias metodológicas docentes

$\mathrm{V} 2$ = Rendimiento académico

$r$ = relación de variable o correlación

\section{Población}

La población estuvo conformada por 14 docentes y 400 estudiantes de las instituciones educativas públicas representativas de nivel inicial (IEI 525 Reyna del Carmen, IE 652-29 Santa Rosa e IEl 516 Villa María) de la UGEL 1 de San Juan de Miraflores.

\section{Muestra}

En la presente investigación se realizó el muestreo censal, puesto que la muestra fue constituida por la totalidad de la población, es decir, el $100 \%$ respecto a las docentes que por ser finita es de fácil acceso y uso por la investigadora. Por otro lado, se consideró a 150 estudiantes (50 estudiantes por cada institución educativa) para el recojo de las calificaciones en su rendimiento académico anual del área de Comunicación.

Se aplicaron dos instrumentos a las docentes. Por un lado, una ficha de observación. La investigadora estuvo presente en las sesiones de aprendizaje y así se pudo observar lo que desarrolló la docente dentro del aula. 
Esta ficha tiene una estructura de 23 ítems que evaluaron la ejecución de las estrategias metodológicas por las docentes, con base en una escala valorativa que calificó aspectos claves. Por otro lado, una encuesta. Fue una autoevaluación de las 14 docentes para identificar las estrategias metodológicas que suelen desarrollar en una sesión de clases. En esta encuesta se recogen las estrategias que aplicaron las docentes. Se decidió por este cuestionario con la técnica de la encuesta a través de 58 ítems. Cada pregunta que respondió la docente estuvo estructurada con una escala de Likert de frecuencias desde «siempre» hasta «nunca».

La ficha de observación y la encuesta fueron adaptadas del instrumento elaborado por Riva en su investigación de 2016. Ambas fueron elaboradas considerando estrictamente el análisis de contenido para cada dimensión de las variables y se aplicó tomando en cuenta la técnica de la observación y de la encuesta, respectivamente.

\section{Resultados}

En el gráfico 1 se muestra que el rendimiento académico en el área de Comunicación tiene el $28,6 \%$ y está en el nivel de inicio; $35,7 \%$, en proceso, y $35,7 \%$, en logro esperado.

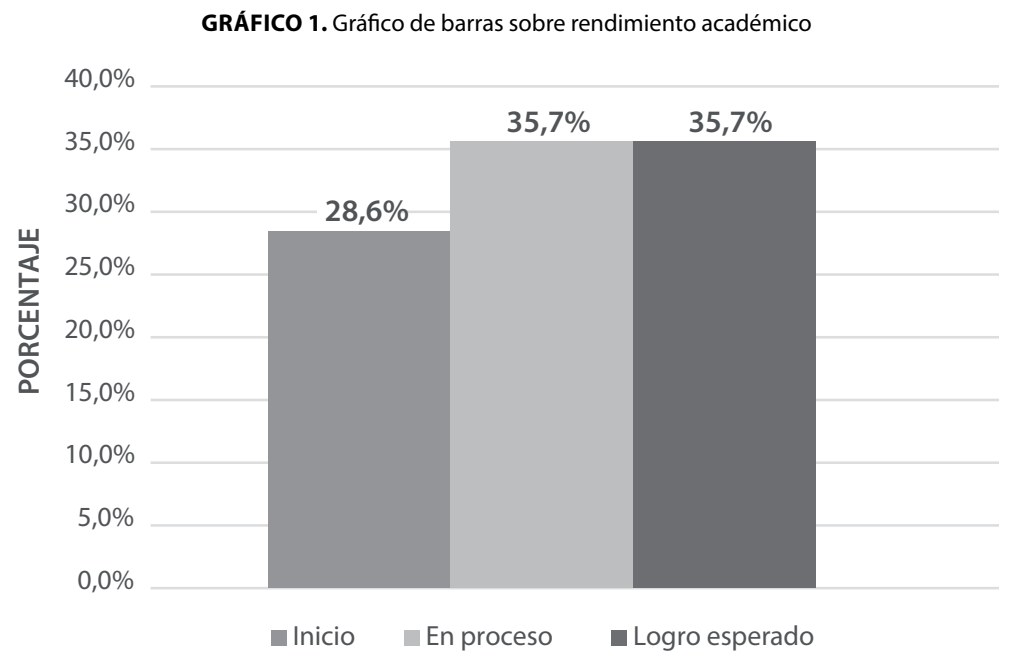

Fuente: Elaboración propia

En el gráfico 2 se muestra que las estrategias metodológicas en la comprensión lectora tienen un $28,6 \%$ y están en el nivel en proceso; $57,1 \%$, en logro esperado, y $14,3 \%$, en logro destacado. 
GRÁFICO 2. Gráfico de barras sobre estrategias metodológicas

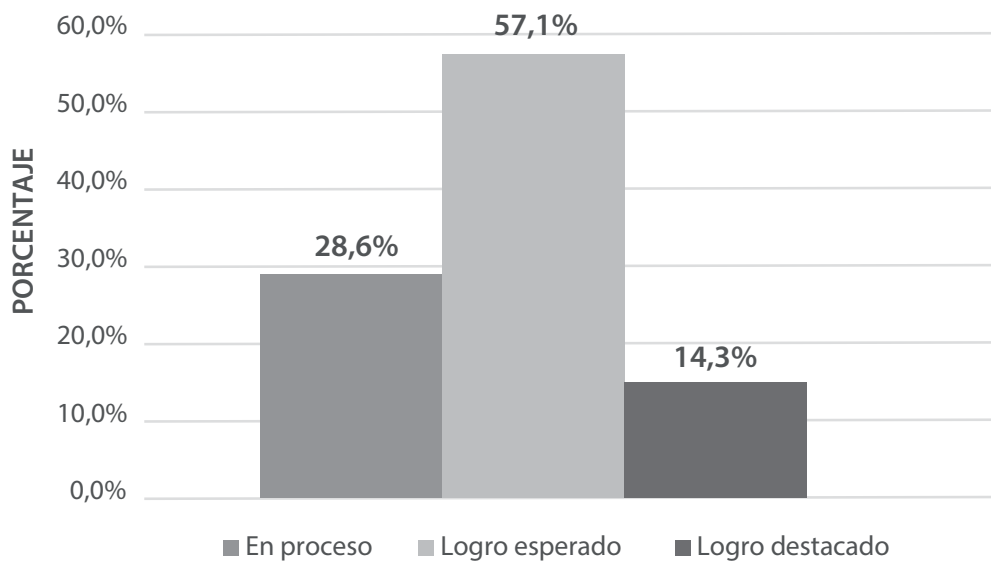

Fuente: Elaboración propia

En el gráfico 3 se muestra que los juegos de lenguaje en la comprensión lectora tienen el $28,6 \%$ y están en el nivel en proceso; $57,1 \%$, en logro esperado, y $14,3 \%$, en logro destacado.

GRÁFICO 3. Gráfico de barras sobre juegos de lenguaje

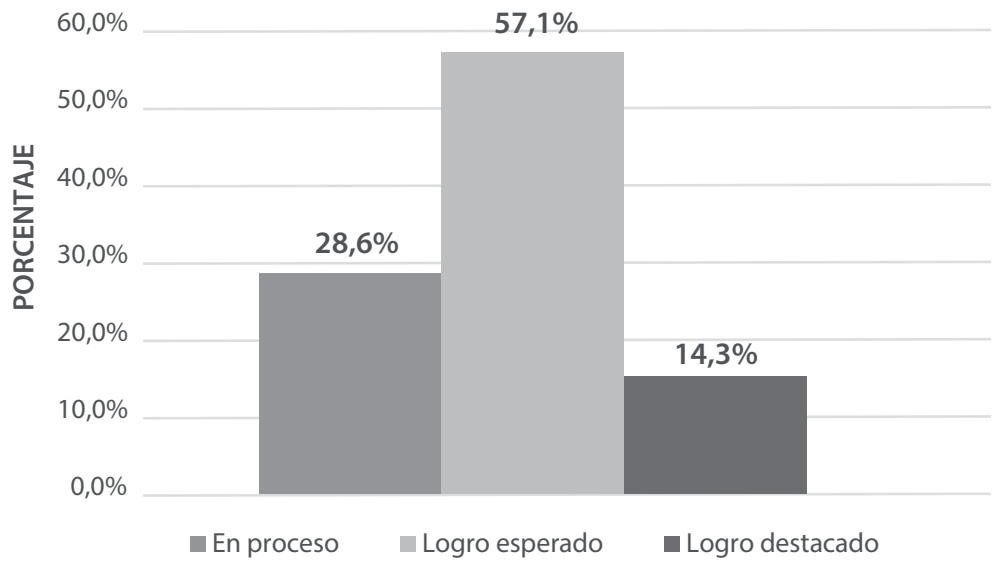

Fuente: Elaboración propia

En el gráfico 4 se muestra que la lectura grupal y en voz alta de cuentos infantiles tiene $14,3 \%$ y está en el nivel inicio; $21,4 \%$, en proceso; $50 \%$, en logro esperado, y $14,3 \%$, en logro destacado. 
GRÁFICO 4. Gráfico de barras sobre lectura grupal y en voz alta de cuentos infantiles (agrupado)

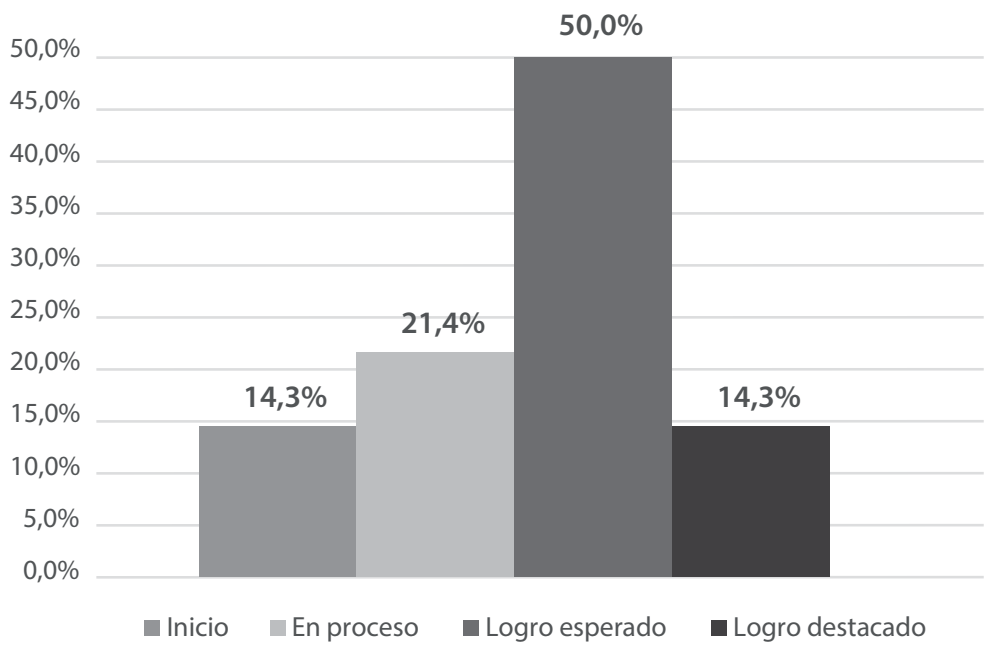

Fuente: Elaboración propia

En el gráfico 5 , se muestra que el teatrino tiene $28,6 \%$ y está en el nivel inicio; $28,6 \%$, en proceso; $35,7 \%$, en logro esperado, y $7,1 \%$, en logro destacado.

GRÁFICO 5. Gráfico de barras sobre teatrino

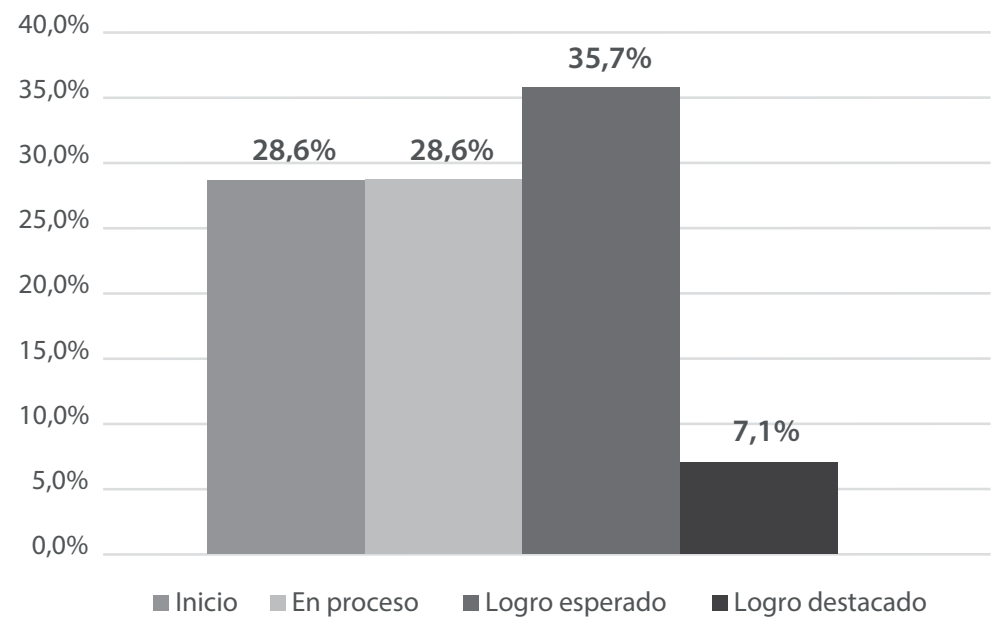

Fuente: Elaboración propia 
En el gráfico 6 se muestra que observación-descripción-creación tiene $14,3 \%$ y está en el nivel de inicio; $28,6 \%$, en proceso, y $57,1 \%$, logro esperado.

GRÁFICO 6. Gráfico de barras sobre observación-descripción-creación

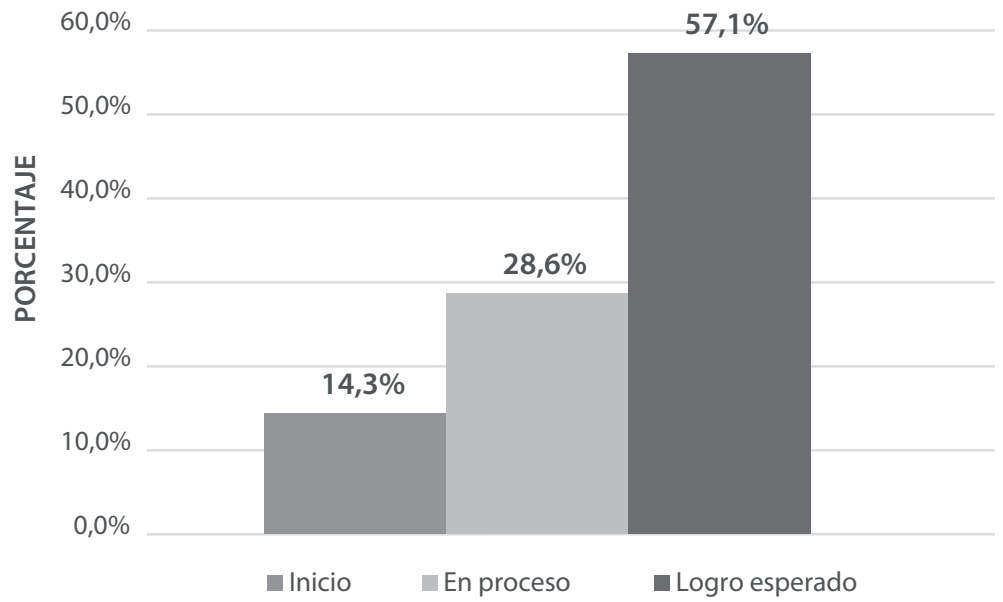

Fuente: Elaboración propia

En el gráfico 7 , se muestra que la poesía tiene $21,4 \%$ y está en el nivel inicio; $42,9 \%$, en proceso; $21,4 \%$, en logro esperado, y $14,3 \%$, en logro destacado.

GRÁFICO 7. Gráfico de barras sobre poesía

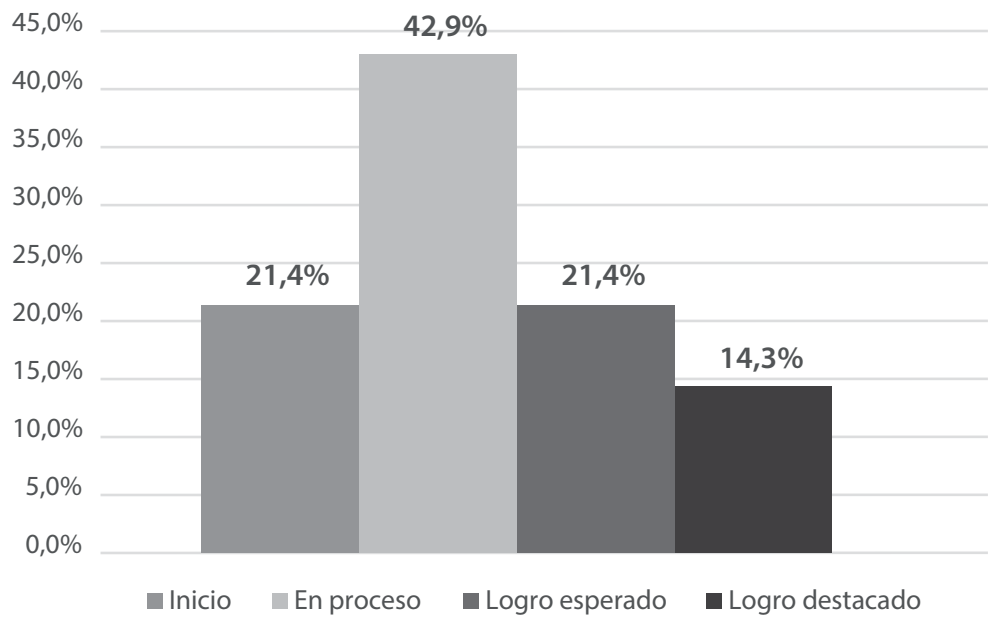

Fuente: Elaboración propia 
En el gráfico 8 se muestra que la expresión-imaginación-creatividad tiene $28,6 \%$ y está en el nivel inicio; $42,9 \%$, en proceso, y $28,6 \%$, en logro destacado.

GRÁFICO 8. Gráfico de barras sobre expresión-imaginación-creatividad

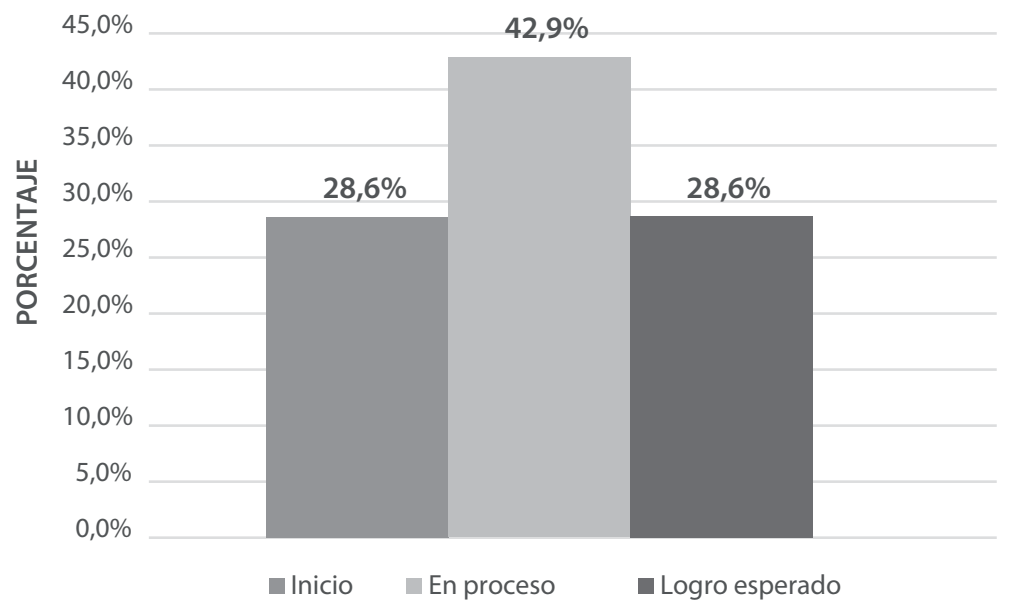

Fuente: Elaboración propia

\section{Discusión}

Las estrategias son un punto clave en los niños preescolares que necesitan una forma de enseñanza didáctica y motivadora. Para ello, se considera el informe de Díaz R. Glorys y Trejo C. Belkis (2007), el cual menciona estrategias metodológicas que se utilizan para iniciar el interés y la motivación del niño hacia la lectura. Ramírez (2018) muestra que la estrategia metodológica se relaciona directa y significativamente con el aprendizaje de los estudiantes de la especialidad de Contabilidad Informática del ISTE Quillabamba, filial Pichari $(p=0,000<0,05$, rho de Sperman $=0,811$, siendo correlación positiva alta). Asimismo, Reyes (2017) enfatizó que las estrategias metodológicas sí influyen sobre el rendimiento académico de la asignatura de matemática básica con un nivel de significancia de 0,00 $=5$. Por otro lado, nuestra investigación resultó que rho de Spearman $r=$ 0,864 y una significancia de $p=0(p<0,05)$. Por lo tanto, afirmamos que existe una relación directa entre las estrategias metodológicas del docente en la comprensión lectora y el rendimiento académico en el área de Comunicación en los estudiantes de 5 años de las instituciones educativas públicas representativas de inicial de la UGEL 1 de San Juan de Miraflores.

Cuando se realizan juegos y actividades, el niño desarrolla y enriquece su lenguaje oral. Todas estas actividades deben ser ejecutadas dentro de 
un contexto significativo y ameno para propiciar el interés y el placer de los niños. Por ello, Padilla (2016) muestra la importancia de talleres para darles a conocer las diversas estrategias metodológicas y respondan a las motivaciones de los estudiantes las cual vemos reflejado en rho de Spearman $r=0,733$ y una significancia de $p=0,003(p<0,05)$. Por consiguiente, afirmamos que existe una relación directa entre los juegos de lenguaje en la comprensión lectora y el rendimiento académico en el área de Comunicación en los estudiantes de 5 años de las instituciones educativas públicas representativas de nivel inicial de la UGEL 1 de San Juan de Miraflores.

La lectura en el nivel de educación inicial debe aplicarse para estimular a los niños y así puedan disfrutar del momento lector. El objetivo de leerles a los niños es que se comparta un momento grato, mas no con el objetivo de evaluarlos o enseñarles algo en particular. Sobre ello, Reyes (2017) refiere que las estrategias metodológicas sí influyen significativamente en la capacidad de colaboración del área de matemática básica. Por lo tanto, comprobamos en este estudio que rho de Spearman $r=0,717$ y tiene una significancia de $p=0,004$ ( $p<0,05)$. Por tanto, afirmamos que existe una relación directa entre la lectura grupal y en voz alta de cuentos infantiles en la comprensión lectora y el rendimiento académico en el área de Comunicación en los estudiantes de 5 años de las instituciones educativas públicas representativas de nivel inicial de la UGEL 1 de San Juan de Miraflores.

El teatrino es un recurso que utiliza el docente al momento de contar un cuento o narrar una historia en particular. Para ello, se utiliza títeres de manos y de dedos o se puede hacer representaciones de animales con sus características e imitación de sus sonidos. Domínguez (2015) expone que existe relación significativa entre las estrategias didácticas y el rendimiento académico de los estudiantes de educación secundaria de las instituciones educativas del distrito de Taurija en La Libertad en 2013. En su estudio observamos similitud con un rho de Spearman $r=0,756$ y una significancia de $p=0,002(p<0,05)$. Por consiguiente, afirmamos que existe una relación directa entre el teatrino en la comprensión lectora y el rendimiento académico en el área de Comunicación en los estudiantes de 5 años de las instituciones educativas públicas representativas de nivel inicial de la UGEL 1 de San Juan de Miraflores.

Los niños tienen que observar, identificar y pronunciar las características. Ya sea en forma, colores, tamaños, de los objetos que puedan observar en el aula como figura de animales, personas representadas en láminas o cuentos, objetos conocidos, entre otros. Valdez (2018) sostiene que existe una relación positiva entre las estrategias metodológicas y la capacidad de análisis de la lectura de los estudiantes del X ciclo de 
educación inicial con un rho de Spearman $r=0,703$, con una $p=0,001$ ( $p$ $<0,005)$, a un nivel de significancia de $5 \%$ estadísticamente significativa. Por lo tanto, se rechazó la hipótesis nula y se aceptó la hipótesis alterna por lo que estamos de acuerdo, ya que obtuvimos rho de Spearman $r=$ 0,817 y una significancia de $p=0(p<0,05)$. Por lo tanto, afirmamos que existe una relación directa entre la observación-descripción-creación en la comprensión lectora y el rendimiento académico en el área de Comunicación en los estudiantes de 5 años de las instituciones educativas públicas representativas de nivel inicial de la UGEL 1 de San Juan de Miraflores.

La poesía puede divertir, motivar y dar placer a los niños de una forma natural. Es una buena vía para acercarlos hacia la lectura. Fernando (2014) induce que guarda relación con la metodología estratégica y los modos de implementar las actividades en grupo, presencial o no presencial, entregable y exposición oral. Se obtuvo mayor resultado y mayor alcance del desarrollo de competencias en los estudiantes titulados al sumar nuestra investigación con rho de Spearman $r=0,827$ y una significancia de $p=$ $0(p<0,05)$. Por consiguiente, afirmamos que existe una relación directa entre la poesía en la comprensión lectora y el rendimiento académico en el área de Comunicación en los estudiantes de 5 años de las instituciones educativas públicas representativas de nivel inicial de la UGEL 1 de San Juan de Miraflores.

Después de analizar cada una de las estrategias, Avidón (2019) sostiene que existe una incidencia significativa de las estrategias metodológicas de la docencia universitaria en el desarrollo de las competencias de la carrera profesional de administración bancaria en los estudiantes del ISTP Blaise Pascal del distrito de Tarapoto. Así, se evalúa en esta investigación con un rho de Spearman $r=0,944$ y una significancia de $p=0(p<0,05)$. Por lo tanto, afirmamos que existe una relación directa entre la expresiónimaginación-creatividad en la comprensión lectora y el rendimiento académico en el área de Comunicación en los estudiantes de 5 años de las instituciones educativas públicas representativas de nivel inicial de la UGEL 1 de San Juan de Miraflores. 


\section{Contribución del autor}

Rosa Eufemia Choquichanca Lavado y Miguel Gerardo Inga Arias han participado en la elaboración, la compilación de datos, la redacción y el consentimiento de la versión final del presente artículo.

\section{Fuente de financiamiento}

Autofinanciado.

\section{Conflicto de interés}

Los autores declaran no tener compromisos de interés. 


\section{REFERENCIAS BIBLIOGRÁFICAS}

Arellano, M. (2012). La competencia literaria en educación infantil. Estrategias didácticas y materiales literarios como factores de desarrollo (tesis de doctorado). Universidad de Valladolid, Valladolid.

Avidón, M. (2019). Estrategias metodológicas de la docencia universitaria en el desarrollo de las competencias de la carrera profesional Administración Bancaria de los estudiantes del ISTP Blaise Pascal del distrito de Tarapoto (tesis de maestría en Ciencias de la Educación con mención en Docencia Universitaria). Universidad Nacional de Educación Enrique Guzmán y Valle, Lima.

Cáceres, A., Donoso, P. y Guzmán, J. (2012). Comprensión lectora. Significados que le atribuyen las docentes al proceso de comprensión lectora en NB2 (tesis para optar al título de Educadora de Párvulos y Escolares Iniciales). Universidad de Chile, Santiago de Chile.

Condemarín, M. (1982). El poder de leer. Recuperado de http://es.scribd. com/doc/2887316/El-Poder-de-Leer

Díaz, G. y Trejo, B. (2007). Estrategias didácticas para promover el placer por la lectura en el aula de preescolar (tesis de licenciatura en Educación con mención Preescolar). Universidad de los Andes, Mérida. Recuperado de https://es.scribd.com/document/332308258/Diaz-y-Belkis-unlocked

Domínguez, R. (2015). Estrategias didácticas y rendimiento académico de los estudiantes de Educación secundaria de las instituciones educativas del distrito de Taurija, La Libertad, 2013 (tesis de magíster en Ciencias de la Educación con mención en Gestión Educacional). Universidad Nacional de Educación Enrique Guzmán y Valle, Lima.

Fernando, L. (2014). Estudio y análisis de metodologías docentes adecuadas para el desarrollo de competencias genéricas en los títulos de grado de Ingeniería Industrial (tesis de doctorado). Universidad de Valladolid, Valladolid.

Matesanz, M. (2013). La lectura en la educación primaria: marco teórico y propuesta de intervención. Recuperado de https://uvadoc.uva.es/bitstream/10324/1474/1/TFG-B.97.pdf

Mejía, E. (2013). La investigación científica en educación. Lima: Universidad Nacional Mayor de San Marcos. Recuperado de https://goo.gl/h7IVUz

Ministerio de Educación del Perú (2017). Reporte de cumplimiento misional 2013-2016. Informe para la Transferencia de Gestión. Lima: Ministerio de Educación del Perú.

Morán, A. y Uzcátegui, A. (2006). Estrategias para el desarrollo de la comprensión lectora de los estudiantes del octavo grado de educación básica. Revista de Artes y Humanidades Única, 7(16), pp. 35-55. Recuperado de http://www.redalyc.org/pdf/1701/170118726003.pdf 
Padilla, J. (2016). Influencia de la estrategia metodológica COP en la comprensión lectora de estudiantes de la especialidad de educación en la Universidad nacional Pedro Ruiz Gallo, Chiclayo. 2015 (tesis de maestría en Educación con mención en Docencia en el Nivel Superior). Universidad Nacional Mayor de San Marcos, Lima.

Perrenoud, P. (2004). Diez nuevas competencias para enseñar. Barcelona: Graó.

Pozo, J. (2008). Aprendices y maestros. Madrid: Alianza Editorial.

Ramírez, A. (2018). Estrategias metodológicas del docente y el aprendizaje de los estudiantes de la especialidad Contabilidad-Informática del ISTE QuiIlabamba Filial Pichari, Cusco 2017 (tesis de maestría en Ciencias de la Educación con mención en Docencia Universitaria). Universidad Nacional de Educación Enrique Guzmán y Valle, Lima.

Reyes, K. (2017). Las estrategias metodológicas del docente y su influencia en el logro de las competencias del curso Matemática Básica de los alumnos del primer ciclo de la Universidad Nacional de Cañete (tesis de maestría en Ciencias de la Educación con mención en Docencia Universitaria). Universidad Nacional de Educación Enrique Guzmán y Valle, Lima.

Rojas, A. (2000). La lectura-escritura en la edad preescolar. Congreso Mundial de Lecto-escritura en Valencia. Recuperado de www.oei.es/historico/ inicial/articulos/lecto_escritura_preescolar.pdf

Rojas, M. (2017). Estrategias cognitivas y los niveles de comprensión lectora en los estudiantes del VI ciclo del nivel secundaria de la Institución Educativa nro. 6081 Manuel Scorza, Torres de San Gabriel Alto en Villa María del Triunfo (tesis de magíster en Educación con mención en Didáctica de la Comunicación en la Educación Básica). Recuperado de http:// cybertesis.unmsm.edu.pe/bitstream/handle/cybertesis/7020/Rojas_ sm.pdf? sequence $=1$ \&isAllowed $=y$

Rojas, M. (2018). La influencia del chat en la escritura de los estudiantes. Desde el Sur, 9(2), pp. 433-445. Recuperado de https://revistas.cientifica. edu.pe/index.php/desdeelsur/article/view/367

Solé, I. (2011). Competencia lectora y aprendizaje. Barcelona: Ediciones de la Universidad de Barcelona. 